\title{
ON THE SEMIGROUP OF ORDER-DECREASING PARTIAL ISOMETRIES OF A FINITE CHAIN
}

\section{R. KEHINDE ${ }^{1}$, S. O. MAKANJUOLA ${ }^{2}$ and A. UMAR ${ }^{3}$}

${ }^{1}$ Department of Mathematics and Statistics

Bowen University

P. M. B. 284, Iwo

Osun State

Nigeria

e-mail: kennyrot2000@yahoo.com

2Department of Mathematics

University of Ilorin

P. M. B. 1515, Ilorin

Kwara State

Nigeria

e-mail: somakanjuola@unilorin.edu.ng

${ }^{3}$ Department of Mathematics

Petroleum Institute

Khalifa University of Science and Technology

Sas Nakhl, P. O. Box 2533

Abu Dhabi

U. A. E.

e-mail: aumar@pi.ac.ae

2010 Mathematics Subject Classification: 20M18, 20M20.

Keywords and phrases: partial one-to-one transformation, partial isometries, height, right (left) waist, right (left) shoulder and fix of a transformation, translations, reflections and idempotents rank of a semigroup.

Financial support from Sultan Qaboos University Internal Grant: IG/SCI/DOMS/13/06 is gratefully acknowledged.

Received December 1, 2017

(C) 2018 Scientific Advances Publishers 


\begin{abstract}
Let $\mathcal{I}_{n}$ be the symmetric inverse semigroup on $X_{n}=\{1,2, \ldots, n\}$ and let $\mathcal{D D P}_{n}$ and $\mathcal{O D D P}_{n}$ be its subsemigroups of order-decreasing partial isometries and of order-preserving and order-decreasing partial isometries of $X_{n}$, respectively. In this paper, we investigate the cycle structure of an orderdecreasing partial isometry and characterize the starred Green's relations on $\mathcal{D D P}_{n}$ and $\mathcal{O D D P}_{n}$. We show that $\mathcal{D D P}_{n}$ is an ample semigroup and $\mathcal{O D D P}_{n}$ is a 0 -E-unitary ample semigroup. We also investigate the ranks of $\mathcal{D D P}{ }_{n}$ and $\mathcal{O D D P}_{n}$
\end{abstract}

\title{
1. Introduction and Preliminaries
}

Let $X_{n}=\{1,2, \ldots, n\}$ and $\mathcal{I}_{n}$ be the symmetric inverse semigroup (consisting of all partial one-to-one transformations of $X_{n}$ ) under composition of mappings. The symmetric inverse semigroup $\mathcal{I}_{n}$ is indeed an inverse semigroup (that is, for all $\alpha \in \mathcal{I}_{n}$ there exists a unique $\alpha^{\prime} \in \mathcal{I}_{n}$ such that $\alpha=\alpha \alpha^{\prime} \alpha$ and $\alpha^{\prime}=\alpha^{\prime} \alpha \alpha^{\prime}$ ). Inverse semigroups (see [14], Chapter V and [15]) are of interest not only as a naturally occurring special case of semigroups but also for their role in describing partial symmetries. Mathematically this property is expressed by the VagnerPreston Theorem ([14], Theorem V.I.10), by which every (finite) inverse semigroup is embedded in an appropriate (finite) symmetric inverse semigroup $\mathcal{I}_{n}$. Every finite inverse semigroup $S$ is embeddable in $\mathcal{I}_{n}$, the analogue of Cayley's theorem for finite groups. Thus, just as the study of symmetric, alternating and dihedral groups has made a significant contribution to group theory, so has the study of various subsemigroups of $\mathcal{I}_{n}$, see, for example, [3, 7, 10, 11, 17, 18, 19].

A transformation $\alpha \in \mathcal{I}_{n}$ is said to be a partial isometry if (for all $x, y \in \operatorname{Dom} \alpha) \quad|x-y|=|x \alpha-y \alpha|$; order-preserving (order-reversing) if (for all $x, y \in \operatorname{Dom} \alpha) x \leq y \Rightarrow x \alpha \leq y \alpha(x \alpha \geq y \alpha)$; and, order-decreasing (order-increasing) if (for all $x \in \operatorname{Dom} \alpha) x \alpha \leq x(x \alpha \geq x)$. Semigroups of partial isometries on more restrictive but richer mathematical structures have been studied [4, 20]. Al-Kharousi et al. [1] initiated a general study 
of the semigroup of partial isometries of $X_{n}, \mathcal{D P} \mathcal{P}_{n}$ and its subsemigroup of order-preserving partial isometries $\mathcal{O D P}_{n}$. Earlier, one of the authors studied the semigroup of partial one-to-one order-decreasing (orderincreasing) transformations of $X_{n}, \mathcal{I}_{n}^{-}$[18] $\left(\mathcal{I}^{+}(X)\right.$ [19]), respectively. Analogous to Al-Kharousi et al. [1] this paper investigates the algebraic and rank properties of $\mathcal{D D P}{ }_{n}$ and $\mathcal{O D D P}_{n}$, the semigroups of orderdecreasing partial isometries and of order-preserving and orderdecreasing partial isometries of $X_{n}$, respectively. Thus, this paper has more or less the same format as Al-Kharousi et al. [1].

In this section, we introduce basic terminology and preliminary results concerning the cycle structure of a partial order-decreasing isometry of $X_{n}$. In the next section (Section 2), we characterize the classical Green's relations and their starred analogues, where we show in contrast to the oversemigroups $\mathcal{D P}_{n}$ and $\mathcal{O D P}_{n}$ that $\mathcal{O D D P}_{n}$ and $\mathcal{D D P}{ }_{n}$ are non-regular. However, we show that $\mathcal{D D P}{ }_{n}$ is a (non-regular) ample semigroup and $\mathcal{O D D P}_{n}$ is a (non-regular) 0-E-unitary ample semigroup. Ample semigroups (formerly known as type A) were introduced by Fountain [8] as certain generalizations of inverse semigroups.

For standard concepts in semigroup and symmetric inverse semigroup theory, see, for example, [14, 17, 15]. In particular, $E(S)$ denotes the set of idempotents of $S$ and $i d_{A}$ denotes the partial identity of the set $A$. Let

$$
\mathcal{D D P}_{n}=\left\{\alpha \in \mathcal{D P}_{n}:(\forall x \in \operatorname{Dom} \alpha) x \alpha \leq x\right\} .
$$

be the subsemigroup of $\mathcal{I}_{n}$ consisting of all order-decreasing partial isometries of $X_{n}$. Also let

$$
\mathcal{O D D P}_{n}=\left\{\alpha \in \mathcal{D D P}_{n}:(\forall x, y \in \operatorname{Dom} \alpha) x \leq y \Rightarrow x \alpha \leq y \alpha\right\}
$$

be the subsemigroup of $\mathcal{D D P}_{n}$ consisting of all order-preserving and order-decreasing partial isometries of $X_{n}$. Then we have the following result. 
Lemma 1.1. $\mathcal{D D P}_{n}$ and $\mathcal{O D D P}_{n}$ are subsemigroups of $\mathcal{I}_{n}$.

Remark 1.2. $\mathcal{D D P}_{n}=\mathcal{D P}_{n} \cap \mathcal{I}_{n}^{-} \quad$ and $\quad \mathcal{O D D P}_{n}=\mathcal{O D P}_{n} \cap \mathcal{I}_{n}^{-}$, where $\mathcal{I}_{n}^{-}$is the semigroup of partial one-to-one order-decreasing transformations of $X_{n}$ [18].

As remarked in [1], it is straightforward that a partial isometry of $X_{n}$ is determined by its domain and the image of two different points of $X_{n}$. Equivalently, it is determined by its domain, the image of a single point and by the knowledge of whether it is order-preserving or orderreversing. As a consequence, elements of any subset of $\mathcal{D P} \mathcal{P}_{n}$ fall into two categories: translations and reflections (about a point or midpoint of two points of $X_{n}$ ). It is also clear that nonidempotent translations do not have fixed points and reflections can have at most one fixed point. Now, let $\alpha$ be an arbitrary element in $\mathcal{I}_{n}$. The height or rank of $\alpha$ is $h(\alpha)=$ $|\operatorname{Im} \alpha|$, the right $[$ left $]$ waist of $\alpha$ is $w^{+}(\alpha)=\max (\operatorname{Im} \alpha),\left[w^{-}(\alpha)=\min \right.$ $(\operatorname{Im} \alpha)]$, the right [left] shoulder of $\alpha$ is $\varpi^{+}(\alpha)=\max (\operatorname{Dom} \alpha)$ $[\varpi(\alpha)=\min (\operatorname{Dom} \alpha)]$, and fix of $\alpha$ is denoted by $f(\alpha)$, and defined by $f(\alpha)=|F(\alpha)|$, where

$$
F(\alpha)=\left\{x \in X_{n}: x \alpha=x\right\}
$$

Next we quote some parts of ([1], Lemma 1.2) that will be needed as well as state an additional observation that will help us understand more the cycle structure of order-decreasing partial isometries.

Lemma 1.3. Let $\alpha \in \mathcal{D P}{ }_{n}$. Then we have the following:

(a) The map $\alpha$ is either order-preserving or order-reversing. Equivalently, $\alpha$ is either a translation or a reflection.

(b) If $\alpha$ is order-preserving and $f(\alpha) \geq 1$, then $\alpha$ is a partial identity.

(c) If $\alpha$ is order-decreasing and $F(\alpha)=\{i\}$, then Dom $\alpha \subseteq\{i, i+1$, $\ldots, n\}$. 
Lemma 1.4 ([18], Lemma 2.1). Let $\alpha$ and $\beta$ be elements in $\mathcal{I}_{n}^{-}$. Then $F(\alpha \beta)=F(\alpha) \cap F(\beta)$.

\section{Green's Relations and their Starred Analogues}

For the definitions of Green's relations, we refer the reader to Howie ([14], Chapter 2). First we have

Theorem 2.1. Let $\mathcal{D D P}_{n}$ and $\mathcal{O D D P}_{n}$ be as defined in (1) and (2), respectively. Then $\mathcal{D D P}_{n}$ and $\mathcal{O D D P}_{n}$ are $\mathcal{J}$-trivial.

Proof. It follows from ([18], Lemma 2.2) and Remark 1.2.

Now since $\mathcal{O D D P}_{n},(n>1)$ contains some nonidempotent elements:

$$
\left(\begin{array}{l}
x \\
y
\end{array}\right)(x>y \geq 1),
$$

it follows immediately that

Corollary 2.2. For $n>1, \mathcal{D D P}_{n}$ and $\mathcal{O D D P}_{n}$ are non-regular semigroups.

On the semigroup $S$ the relation $\mathcal{L}^{*}\left(\mathcal{R}^{*}\right)$ is defined by the rule that $(a, b) \in \mathcal{L}^{*}\left(\mathcal{R}^{*}\right)$ if and only if the elements $a, b$ are related by the Green's relation $\mathcal{L}(\mathcal{R})$ in some oversemigroup of $S$. The join of the equivalences $\mathcal{L}^{*}$ and $\mathcal{R}^{*}$ is denoted by $\mathcal{D}^{*}$ and their intersection by $\mathcal{H}^{*}$. For the definition of the starred analogue of the Green's relation $\mathcal{J}$, see [9] or [18]. Note that on any regular (inverse) semigroup the starred relations coincide with the (corresponding) usual Green's relations [9].

A semigroup $S$ in which each $\mathcal{L}^{*}$-class and each $\mathcal{R}^{*}$-class contains an idempotent is called abundant [9].

By ([6], Lemma 1.6) and ([14], Proposition 2.4.2 \& Ex. 5.11.2), we deduce the following lemma: 
Lemma 2.3. Let $\alpha, \beta \in \mathcal{D D P}_{n}$ or $\mathcal{O D D P}_{n}$. Then

(1) $\alpha \leq_{\mathcal{R}^{*}} \beta$ if and only if $\operatorname{Dom} \alpha \subseteq \operatorname{Dom} \beta$;

(2) $\alpha \leq_{\mathcal{L}^{*}} \beta$ if and only if $\operatorname{Im} \alpha \subseteq \operatorname{Im} \beta$;

(3) $\alpha \leq_{\mathcal{H}^{*}} \beta$ if and only if $\operatorname{Dom} \alpha \subseteq \operatorname{Dom} \beta$ and $\operatorname{Im} \alpha \subseteq \operatorname{Im} \beta$.

Proof. It is enough to observe that $\mathcal{O D D P}_{n}$ and $\mathcal{D D P} \mathcal{P}_{n}$ are full subsemigroups of $\mathcal{I}_{n}$ in the sense that $E\left(\mathcal{O D D P}_{n}\right)=E\left(\mathcal{D D P} \mathcal{P}_{n}\right)=E\left(\mathcal{I}_{n}\right)$.

As in Al-Kharousi et al. [1] to characterize the analogue of Green's relation $\mathcal{D}$, first we define the gap and reverse gap of the image set of $\alpha$ as ordered $(p-1)$-tuples as follows:

$$
g(\operatorname{Im} \alpha)=\left(\left|a_{2} \alpha-a_{1} \alpha\right|,\left|a_{3} \alpha-a_{2} \alpha\right|, \ldots,\left|a_{p} \alpha-a_{p-1} \alpha\right|\right),
$$

and

$$
\left.g^{R}(\operatorname{Im} \alpha)=\left(\left|a_{p} \alpha-a_{p-1} \alpha\right|\right), \ldots,\left|a_{3} \alpha-a_{2} \alpha\right|,\left|a_{2} \alpha-a_{1} \alpha\right|\right)
$$

where $\alpha=\left(\begin{array}{cccc}a_{1} & a_{2} & \cdots & a_{p} \\ a_{1} \alpha & a_{2} \alpha & \cdots & a_{p} \alpha\end{array}\right)$ with $1 \leq a_{1}<a_{2}<\cdots<a_{p} \leq n$.

For example, if

$$
\alpha=\left(\begin{array}{ccccc}
1 & 2 & 4 & 7 & 8 \\
3 & 4 & 6 & 9 & 10
\end{array}\right), \beta=\left(\begin{array}{cccc}
2 & 4 & 7 & 8 \\
10 & 8 & 5 & 4
\end{array}\right) \in \mathcal{D P}_{10}
$$

then $g(\operatorname{Im} \alpha)=(1,2,3,1), g(\operatorname{Im} \beta)=(2,3,1), g^{R}(\operatorname{Im} \alpha)=(1,3,2,1)$, and $g^{R}(\operatorname{Im} \beta)=(1,3,2)$. From [1], we have

Lemma 2.4 ([1], Lemma 2.3). Let $\alpha, \beta \in \mathcal{D P}{ }_{n}$. Then $g(\operatorname{Im} \alpha)=g(\operatorname{Im} \beta)$ or $g(\operatorname{Im} \alpha)=g^{R}(\operatorname{Im} \beta)$ if and only if there is an isometry between $\operatorname{Im} \alpha$ and $\operatorname{Im} \beta$. 
However, in $\mathcal{D D P}_{n}$ things are not as smooth as in $\mathcal{D P}{ }_{n}$. The next theorem gives a characterization of $\mathcal{D}^{*}$ in $\mathcal{D D P}_{n}$.

Theorem 2.5. Let $S=\mathcal{D D P}_{n}$. Suppose

$$
\alpha=\left(\begin{array}{cccc}
a_{1} & a_{2} & \ldots & a_{p} \\
a_{1} \alpha & a_{2} \alpha & \ldots & a_{p} \alpha
\end{array}\right) \text { and } \beta=\left(\begin{array}{cccc}
b_{1} & b_{2} & \ldots & b_{p} \\
b_{1} \alpha & b_{2} \alpha & \ldots & b_{p} \alpha
\end{array}\right)
$$

are elements in $\mathcal{D D P}_{n}$ with $1 \leq a_{1}<a_{2}<\cdots<a_{p} \leq n$ and $1 \leq b_{1}<b_{2}$ $<\cdots<b_{p} \leq n$. Then $(\alpha, \beta) \in \mathcal{D}^{*}$ if and only if there exists an orderpreserving isometry $\theta: \operatorname{Dom} \alpha \rightarrow \operatorname{Dom} \beta ;$ or there exists an orderreversing isometry $\theta^{\prime}: \operatorname{Im} \alpha \rightarrow \operatorname{Im} \beta$ and $(n-1) / 2 \geq a_{p}-a_{1}=b_{p}-b_{1}$ for $n \geq 7$. Moreover, for $2 \leq n \leq 6$ we have $\mathcal{D}^{*}=\mathcal{R}^{*} \circ \mathcal{L}^{*} \circ \mathcal{R}^{*}=\mathcal{L}^{*} \circ$ $\mathcal{R}^{*} \circ \mathcal{L}^{*}$, and for $n \geq 7$ we have $\mathcal{D}^{*}=\left(\mathcal{R}^{*} \circ \mathcal{L}^{*}\right)^{2}=\left(\mathcal{L}^{*} \circ \mathcal{R}^{*}\right)^{2}$.

Proof. Case 1. Define $\theta: \operatorname{Dom} \alpha \rightarrow \operatorname{Dom} \beta$ by $\left(a_{i}\right) \theta=b_{i}$. Then $\theta$ is an order-preserving isometry, and we may without loss of generality suppose $a_{1} \geq b_{1}$. Then by isometry and order-preserving properties we must have $a_{i} \geq b_{i}$ for all $i \in\{1,2, \ldots, p\}$. It is now not difficult to see that $\theta \beta \in \mathcal{D D P}_{n}$ and $\alpha \mathcal{R}^{*} \theta \beta \mathcal{L}^{*} \beta$ whence $(\alpha, \beta) \in \mathcal{D}^{*}$. Notice that $i d_{\{1\}} \mathcal{L}\left(\begin{array}{l}2 \\ 1\end{array}\right) \mathcal{R i d}_{\{2\}}$ but there is no $\alpha \in \mathcal{D D P}_{n}$ such that $i d_{\{1\}} \mathcal{R} \alpha \mathcal{L} i d_{\{2\}}$. Thus, $\mathcal{R}^{*} \circ \mathcal{L}^{*} \neq \mathcal{L}^{*} \circ \mathcal{R}^{*}$.

Case 2. Define $\theta: \operatorname{Dom} \alpha \rightarrow \operatorname{Dom} \beta$ by $\left(a_{p-i+1}\right) \theta^{\prime}=b_{i}$. Then $\theta^{\prime}$ is an order-reversing isometry, and suppose also that $(n-1) / 2 \geq a_{p}-a_{1}$. Further, for $i \in\{1,2, \ldots, p\}$ define the following maps:

$$
\alpha^{\prime}=\left(\begin{array}{c}
n-a_{p}+a_{i} \\
a_{i} \alpha
\end{array}\right), \delta=\left(\begin{array}{c}
n-a_{p}+a_{i} \\
a_{p}-a_{i}+1
\end{array}\right) \text {, and } \beta^{\prime}=\left(\begin{array}{c}
b_{i} \\
a_{p}-a_{p-i+1}+1
\end{array}\right) \text {. }
$$


(i) Since $n-a_{p}+a_{i} \geq a_{i} \geq a_{i} \alpha$, it follows that $\alpha^{\prime}$ is orderdecreasing. Moreover, $\left|\left(n-a_{p}+a_{i}\right) \alpha^{\prime}-\left(n-a_{p}+a_{j}\right) \alpha^{\prime}\right|=\left|a_{i} \alpha-a_{j} \alpha\right|=$ $\left|a_{i}-a_{j}\right|=\left|\left(n-a_{p}+a_{i}\right)-\left(n-a_{p}+a_{j}\right)\right|$, and so $\alpha^{\prime}$ is an isometry.

(ii) First observe that $b_{p}-b_{1}=a_{p}-a_{1}$, and since $n-a_{p}+a_{1}=n$ $-\left(a_{p}-a_{1}\right)=n-\left(b_{p}-b_{1}\right)=n-b_{p}+b_{1} \geq a_{p}-a_{1}+1 \Rightarrow n-\left(a_{p}-a_{i}\right) \geq$ $a_{p}-a_{i}+1$ (for all $i$ ), it follows that $\delta$ is order-decreasing. Moreover, $\left|\left(n-a_{p}+a_{i}\right) \delta-\left(n-a_{p}+a_{j}\right) \delta\right|=\left|\left(a_{p}-a_{i}+1\right)-\left(a_{p}-a_{j}+1\right)\right|=\left|a_{i}-a_{j}\right|=$ $\left|\left(n-a_{p}+a_{i}\right)-\left(n-a_{p}+a_{j}\right)\right|$, and so $\delta$ is an isometry.

(iii) Since $b_{1} \geq 1=a_{p}-a_{p-1+1}+1 \Rightarrow b_{i} \geq a_{p}-a_{p-i+1}+1$ (for all $i$ ), it follows that $\beta^{\prime}$ is order-decreasing. Moreover, $\left|b_{i} \beta^{\prime}-b_{j} \beta^{\prime}\right|=\mid\left(a_{p}-\right.$ $\left.a_{p-i+1}+1\right)-\left(a_{p}-a_{p-j+1}+1\right)|=| a_{p-i+1}-a_{p-j+1}|=|\left(b_{i}\right)\left(\theta^{\prime}\right)^{-1}-\left(b_{j}\right)\left(\theta^{\prime}\right)^{-1} \mid$ $=\left|b_{i}-b_{j}\right|$, and so $\beta^{\prime}$ is an isometry.

Now observe that $\alpha \mathcal{L}^{*} \alpha^{\prime} \mathcal{R}^{*} \delta \mathcal{L}^{*} \beta^{\prime} \mathcal{R}^{*} \beta$ by Lemma 2.3 , and so $\alpha \mathcal{D}^{*} \beta$.

Similarly, we can show that $\alpha \mathcal{R}^{*} \alpha^{\prime \prime} \mathcal{L}^{*} \delta^{\prime} \mathcal{R}^{*} \beta^{\prime \prime} \mathcal{L}^{*} \beta$ for some $\alpha^{\prime \prime}, \delta^{\prime}$, and $\beta^{\prime \prime}$ in $\mathcal{D D P}_{n}$.

Finally, to show that $\mathcal{D}^{*}=\left(\mathcal{R}^{*} \circ \mathcal{L}^{*}\right)^{2}=\left(\mathcal{L}^{*} \circ \mathcal{R}^{*}\right)^{2}$, it is enough to show that $\mathcal{L}^{*} \circ \mathcal{R}^{*} \circ \mathcal{L}^{*} \neq \mathcal{D}^{*} \neq \mathcal{R}^{*} \circ \mathcal{L}^{*} \circ \mathcal{R}^{*}$. To see this, for $n \geq 7$, let

$$
\alpha=\left(\begin{array}{lll}
n-3 & n-2 & n \\
n-3 & n-2 & n
\end{array}\right) \text { and } \beta=\left(\begin{array}{lll}
n-3 & n-1 & n \\
n-3 & n-1 & n
\end{array}\right) \text {. }
$$

Now if $\alpha \mathcal{L}^{*} \alpha^{\prime} \mathcal{R}^{*} \beta^{\prime} \mathcal{L}^{*} \beta$, then

$$
\operatorname{Im} \alpha=\operatorname{Im} \alpha^{\prime}=\{n-3, n-2, n\}, \operatorname{Im} \beta^{\prime}=\operatorname{Im} \beta=\{n-3, n-1, n\},
$$

and

$$
\operatorname{Dom} \alpha^{\prime}=\operatorname{Dom} \beta^{\prime} \subseteq\{n-3, n-2, n-1, n\} .
$$


It is not difficult to see that it is impossible to have a domain that will admit the two possible image sets (for $\alpha^{\prime}$ and $\beta^{\prime}$ ). Thus, $(\alpha, \beta) \notin \mathcal{L}^{*}$ $\circ \mathcal{R}^{*} \circ \mathcal{L}^{*}$

Similarly, we can show that $(\alpha, \beta) \notin \mathcal{R}^{*} \circ \mathcal{L}^{*} \circ \mathcal{R}^{*}$.

An immediate consequence of Lemma 2.4 and Theorem 2.5 is that for $\alpha, \beta \in \mathcal{D D P}_{n}$, we have $(\alpha, \beta) \in \mathcal{D}^{*}$ if and only if

$$
g(\operatorname{Im} \alpha)=\left\{\begin{array}{l}
g(\operatorname{Im} \beta) ; \text { or } \\
g^{R}(\operatorname{Im} \beta), a_{p}-a_{1} \leq(n-1) / 2 .
\end{array}\right.
$$

The corresponding result for $\mathcal{O D D P}_{n}$ can be proved similarly, and is in fact easier.

Theorem 2.6. Let $S=\mathcal{O D D P}_{n}$. Then $\alpha \leq_{\mathcal{D}^{*}} \beta$ if and only if there exists an order-preserving isometry $\theta: \operatorname{Im} \alpha \rightarrow \operatorname{Im} \beta . \quad$ Moreover, $\mathcal{D}^{*}=\mathcal{R}^{*} \circ \mathcal{L}^{*} \circ \mathcal{R}^{*}=\mathcal{L}^{*} \circ \mathcal{R}^{*} \circ \mathcal{L}^{*}$.

Similarly, an immediate consequence of Lemma 2.4 and Theorem 2.6 is that for $\alpha, \beta \in \mathcal{D D P}_{n}$

$$
(\alpha, \beta) \in \mathcal{D}^{*} \text { if and only if } g(\operatorname{Im} \alpha)=g(\operatorname{Im} \beta) .
$$

An abundant semigroup $S$ in which $E(S)$ is a semilattice is called adequate [8]. Of course inverse semigroups are adequate since in this case $\mathcal{L}^{*}=\mathcal{L}$ and $\mathcal{R}^{*}=\mathcal{R}$. As in [8], for an element a of an adequate semigroup $S$, the (unique) idempotent in the $\mathcal{L}^{*}$-class $\left(\mathcal{R}^{*}\right.$-class) containing a will be denoted by $a^{*}\left(a^{+}\right)$. An adequate semigroup $S$ is said to be ample if $e a=a(e a)^{*}$ and $a e=(a e)^{+} a$ for all elements a in $S$ and all idempotents $e$ in $S$. Ample semigroups were known as type $A$ semigroups [13]. 
Theorem 2.7. $\mathcal{D D P}_{n}$ and $\mathcal{O D D P}_{n}$ are non-regular ample semigroups.

Proof. The proofs are similar to that of ([18], Theorem 2.6).

Let $E^{\prime}=E \backslash\{0\}$. A semigroup $S$ is said to be 0 - $E$-unitary if $\left(\forall e \in E^{\prime}\right)(\forall s \in S) e s \in E^{\prime} \Rightarrow s \in E$. That is, a full subsemigroup of $\mathcal{I}_{n}$ is 0 - $E$-unitary if and only if only idempotents have fixed points. The structure theorem for 0-E-unitary inverse semigroups was given by Lawson [16] and, Gomes and Howie [12].

Remark 2.8. $\mathcal{O D D P}_{n}$ is a $0-E$-unitary ample subsemigroup of $\mathcal{I}_{n}$.

\section{Rank Properties}

Let $S$ be a semigroup and let $A$ be a subset of $S$. We say that $A$ is a generating set if $\langle A\rangle=S$. The rank of a finite semigroup $S$ is usually denoted and defined by

$$
\operatorname{rank} S=\min \{|A|: A \subset S,\langle A\rangle=S\} .
$$

Rank properties of various semigroups of transformations have been investigated by various authors in recent years, and we draw particular attention to Gomes and Howie [12], Garba [11], Umar [18], Ganyushkin and Mazorchuk [10] and, more recently Al-Kharousi et al. [1].

\subsection{Rank of $\mathcal{O D D P} \mathcal{P}_{n}$}

It has already been observed (Lemma $1.3(\mathrm{a})$ ) that $\mathcal{O D P}_{n}$ consists of translations. Let

$$
\eta=\left(\begin{array}{cccc}
2 & 3 & \cdots & n \\
1 & 2 & \cdots & n-1
\end{array}\right) .
$$

Then it is easy to see that non-identity translations are restrictions of the map $\eta^{i}$. Next, we observe that $S=\mathcal{D D P}_{n}$ or $S=\mathcal{O D D P}_{n}$ is the union of $K_{0}, K_{1}, \cdots, K_{n}$; where $K_{p}=\{\alpha \in S: h(\alpha)=p\}$. Then clearly

$$
K_{n-1}=\left\{\eta, i d_{X_{n} \backslash\{i\}}: 1 \leq i \leq n\right\},
$$

for $S=\mathcal{O D D P}_{n}$ and it generates $\mathcal{O D D P}_{n} \backslash\left\{i d_{X_{n}}\right\}$. 
It is also not difficult to see that $\eta$ is the unique nonidempotent in $K_{n-1}$. Moreover, we have

Lemma 3.1 ([18], Lemma 3.5). Let $\alpha, \beta, \alpha \beta \in \mathcal{O D D P}_{n}$, be each of height $n-1$. Then $\alpha \beta$ is a partial identity if and only if $\alpha=\beta=\alpha \beta$.

Thus we now have

Theorem 3.2. For $n \geq 2$, we have

(a) rank $\left(\mathcal{O D D P}_{n} \backslash\left\{i d_{X_{n}}\right\}\right)=n+1$. Moreover, $K_{n-1}$ is the unique minimum generating set for $\mathcal{O D D P}_{n} \backslash\left\{i_{X_{n}}\right\}$.

(b) rank $\mathcal{O D D P}_{n}=n+2$. Moreover, $K_{n-1} \cup\left\{i d_{X_{n}}\right\}$ is the unique minimum generating set for $\mathcal{O D D P}_{n}$.

Proof. (a) The minimality of $K_{n-1}$ as a generating set for $\mathcal{O D D P}_{n}$ follows from Lemma 3.1 and the remarks preceding it. The main result in Doyen [5] states that: "Any finite $\mathcal{J}$-trivial monoid has a unique minimum generating system." Thus, by Theorem 2.1, we deduce that $K_{n-1}$ is the unique minimum generating set for $\mathcal{O D D P}_{n}$.

(b) It follows directly from (a) above.

\subsection{Rank of $\mathcal{D D D} \mathcal{P}_{n}$}

To find the rank of $\mathcal{D D P}{ }_{n},(n>2)$, we note the following:

(a) the product of two order-reversing transformations is orderpreserving;

(b) the product of order-reversing and order-preserving transformation is order-reversing;

(c) order-decreasing and order-reversing partial isometries exist only for heights less than or equal to $n / 2$.

Thus since order-preserving maps generate themselves only, we need some order-reversing maps to generate $\mathcal{D D P}_{n}$. 
Now, for $1<i<n$, define

$$
\eta_{i}=\left\{\begin{array}{c}
\left(\begin{array}{cccc}
i & i+1 & \cdots & 2 i-1 \\
i & i-1 & \cdots & 1
\end{array}\right), i \leq\left\lceil\frac{n}{2}\right\rceil \\
\left(\begin{array}{cccc}
i & i+1 & \cdots & n \\
i & i-1 & \cdots & 2 i-n
\end{array}\right), i>\left\lceil\frac{n}{2}\right\rceil
\end{array}\right.
$$

It is clear that each $\eta_{i}$ is an order-reversing nonidempotent (for $n>2$ ) partial isometry and

$$
h\left(\eta_{i}\right)= \begin{cases}i, & i \leq\left\lceil\frac{n}{2}\right\rceil ; \\ n-i+1, & i>\left\lceil\frac{n}{2}\right\rceil .\end{cases}
$$

The next lemma is evident.

Lemma 3.3. For $S=\mathcal{O D D P}_{n}$, we have

$$
\left\{\alpha \in \mathcal{D D P}_{n}: h(\alpha)>\lceil n / 2\rceil\right\}=\left\{\alpha \in \mathcal{O D D P}_{n}: h(\alpha)>\lceil n / 2\rceil\right\} .
$$

The next two lemmas are slightly less evident.

Lemma 3.4. Let $\alpha \in \mathcal{D D P}_{n}$ with $F(\alpha)=\{i\}$. Then $h(\alpha) \leq h\left(\eta_{i}\right)$.

Proof. This follows directly from Lemma 1.3(c).

Lemma 3.5. Let $\alpha, \beta \in \mathcal{D D P}_{n}$ be such that $\alpha \beta=\eta_{i}$. Then $\alpha=\eta_{i}$ or $\beta=\eta_{i}$.

Proof. Let $\alpha \beta=\eta_{i}$ then $i \in F(\alpha) \cap F(\beta)$, by Lemma 1.4. Suppose $\alpha$ is order-preserving and $\beta$ is order-reversing then $\alpha$ is a partial identity by Lemma 1.3(b). Moreover, $h(\beta) \leq h\left(\eta_{i}\right)=h(\alpha \beta) \leq h(\beta)$ by Lemma 3.4, hence $\beta=\eta_{i}$ by Lemma 1.3(c). The case when $\alpha$ is order-reversing and $\beta$ is order-preserving, is similar.

From Lemmas 3.3, 3.4, and 3.5, we deduce the next lemma. 
Lemma 3.6. Any generating set for $\mathcal{D D P}_{n}$ must contain $K_{n-1} \cup\left\{\eta_{i}\right.$ $: 1<i<n\}$.

While the above lemma shows the necessity of $K_{n-1} \cup\left\{\eta_{i}: 1<i<n\right\}$ as part of any generating set for $\mathcal{D D P}_{n}$, the next lemma shows its sufficiency.

Lemma 3.7. $\mathcal{D D P}_{n} \backslash\left\{i d_{X_{n}}\right\}=\left\langle K_{n-1} \bigcup\left\{\eta_{i}: 1<i<n\right\}\right\rangle$.

Proof. The result follows from the facts that nonidempotent translations are restrictions of $\eta^{i}$ and reflections are restrictions of $\eta^{i} \eta_{j}$ for $i \geq 0$.

Theorem 3.8. For $n \geq 2$, we have

(a) $\operatorname{rank}\left(\mathcal{D D P}_{n} \backslash\left\{i d_{X_{n}}\right\}\right)=2 n-1$. Moreover, $K_{n-1} \cup\left\{\eta_{i}: 1<i<n\right\}$ is the unique minimum generating set for $\mathcal{O D D P}_{n} \backslash\left\{i d_{X_{n}}\right\}$.

(b) rank $\mathcal{D D P}_{n}=2 n$. Moreover, $K_{n-1} \cup\left\{\eta_{i}: 1<i<n\right\} \cup\left\{i d_{X_{n}}\right\}$ is the unique minimum generating set for $\mathcal{O D D P}_{n}$.

Proof. (a) It follows from Lemmas 3.5, 3.6, and 3.7, the fact that $\left|K_{n-1}\right|=n+1$ and that there are $n-2 \eta_{i}$ s. For uniqueness, see the proof of Theorem 3.2 above.

(b) It follows directly from (a) above.

We conclude with a table of summary of the rank results proved in the paper.

\begin{tabular}{|c|c|c|c|c|}
\hline Semigroup & $\mathcal{O D D P}_{n} \backslash\left\{i d_{X_{n}}\right\}$ & $\mathcal{O D D P}_{n}$ & $\mathcal{D D P}_{n} \backslash\left\{i d_{X_{n}}\right\}$ & $\mathcal{D D P}_{n} n+1$ \\
\hline Rank & $n+1$ & $n+2$ & $2 n-1$ & $2 n$ \\
\hline
\end{tabular}

\section{Acknowledgements}

The first named author would like to thank Bowen University, Iwo and Sultan Qaboos University for their financial support and hospitality, respectively. 


\section{References}

[1] F. Al Kharousi, R. Kehinde and A. Umar, On the semigroup of partial isometries of a finite chain, Comm. Algebra 44(2) (2016), 639-647.

DOI: https://doi.org/10.1080/00927872.2014.984838

[2] F. Al Kharousi, R. Kehinde and A. Umar, Combinatorial results for certain semigroups of order-decreasing partial isometries of a finite chain (Submitted).

[3] D. Borwein, S. Rankin and L. Renner, Enumeration of injective partial transformations, Discrete Math. 73(3) (1989), 291-296.

DOI: https://doi.org/10.1016/0012-365X(89)90272-0

[4] L. Bracci and L. E. Picasso, Representations of semigroups of partial isometries, Bull. Lond. Math. Soc. 39(5) (2007), 792-802.

DOI: https://doi.org/10.1112/blms/bdm059

[5] J. Doyen, Equipotence et unicité de systèmes generateurs minimaux dans certains monoides, Semigroup Forum 28(1) (1984), 341-346.

DOI: https://doi.org/10.1007/BF02572494

[6] A. El-Qallali and J. B. Fountain, Idempotent-connected abundant semigroups, Proc. Roy. Soc. Edinburgh Sect. A: Math. 91(1-2) (1981), 79-90.

DOI: https://doi.org/10.1017/S0308210500012646

[7] V. H. Fernandes, The monoid of all injective orientation-preserving partial transformations on a finite chain, Comm. Algebra 28(7) (2000), 3401-3426.

DOI: https://doi.org/10.1080/00927870008827033

[8] J. B. Fountain, Adequate semigroups, Proc. Edinburgh Math. Soc. 22(2) (1979), 113-125.

DOI: https://doi.org/10.1017/S0013091500016230

[9] J. B. Fountain, Abundant semigroups, Proc. London Math. Soc. 44(1) (1982), 103-129.

DOI: https://doi.org/10.1112/plms/s3-44.1.103

[10] O. Ganyushkin and V. Mazorchuk, Classical Finite Transformation Semigroups: An Introduction, Springer-Verlag, London, 2009.

[11] G. U. Garba, Nilpotents in semigroups of partial one-to-one order-preserving mappings, Semigroup Forum 48(1) (1994), 37-49.

DOI: https://doi.org/10.1007/BF02573652

[12] G. M. S. Gomes and J. M. Howie, A P-theorem for inverse semigroups with zero, Portugal. Math. 53(3) (1996), 257-278. 
[13] V. Gould, Graph expansions of right cancellative monoids, Internat. J. Algebra Comput. 6(6) (1996), 713-733.

DOI: https://doi.org/10.1142/S0218196796000404

[14] J. M. Howie, Fundamentals of semigroup theory, London Mathematical Society Monographs, New Series 12, Oxford Science Publications, The Clarendon Press, Oxford University Press, New York, 1995.

[15] M. V. Lawson, Inverse semigroups, The theory of partial symmetries, World Scientific Publishing Co., Inc., River Edge, NJ, 1998.

[16] M. V. Lawson, The structure of $0-E$-unitary inverse semigroups I: The monoid case, Proc. Edinburgh Math. Soc. 42(3) (1999), 497-520.

DOI: https://doi.org/10.1017/S0013091500020484

[17] S. Lipscomb, Symmetric inverse semigroups mathematical surveys and monographs, 46, Amer. Math. Soc., Providence, R.I., 1996.

[18] A. Umar, On the semigroups of partial one-to-one order-decreasing finite transformations, Proc. Roy. Soc. Edinburgh, Sect. A: Math. 123(2) (1993), 355-363.

DOI: https://doi.org/10.1017/S0308210500025737

[19] A. Umar, On certain infinite semigroups of order-increasing transformations II, Arab. J. Sci. Eng. 28(2A) (2003), 203-210.

[20] Lawrence J. Wallen, Semigroups of partial isometries, Bull. Amer. Math. Soc. 75(4) (1969), 763-764.

DOI: https://doi.org/10.1090/S0002-9904-1969-12278-0 